Onkologie 2013;36:76

Original Articles

Case Reports

Review Article
The Impact of Computed Tomography on Early Glottic Cancer Outcomes Mourad, W.F.; Hu, K.S.; Shourbaji, R.A.; Ishihara, D.; Lin, W.; Kumar, M.; Blakaj, D.M.; Harrison, L.B. (New York, NY)

Safety Evaluation of High Intensity Focused Ultrasound in Patients with Pancreatic Cancer

Wang, K; Zhu, H.; Meng, Z.; Chen, Z.; Lin, J.; Sheng, Y.; Gao, H. (Shanghai)

Everolimus in Metastatic Renal Cell Carcinoma after Failure of Initial Vascular Endothelial Growth Factor Receptor-Tyrosine Kinase Inhibitor (VEGFr-TKI) Therapy: Results of an Interim Analysis of a Non-Interventional Study Bergmann, L. (Frankfurt/M.); Goebell, P.J. (Erlangen); Kube, U. (Chemnitz); Kindler, M. (Berlin); Herrmann, E. (Münster); Janssen, J. (Westerstede); Schmitz, J. (Arnsberg); Weikert, S. (Berlin); Steiner, G. (Meiningen); Jakob, A. (Offenburg); Staehler, D. (München)

Expression of Rac1 and HIF-1a in VEGF in Gastric Carcinoma: Correlation with Angiogenesis and Prognosis

Zhan, H.; Liang, H.; Liu, X.; Deng, J.; Wang, B.; Hao, X. (Tianjin)

Are Somatic Symptoms a Legitimate Part of the Depression Profile in Prostate Cancer Patients?

Sharpley, C.F. (Armidale, New South Wales); Bitsika, V. (Robina, Queensland); Christie, D.H.R. (Tugun/Southport, Queensland)

MicroRNA-21 as an Indicator of Aggressive Phenotype in Breast Cancer Ozgun, A.; Karagoz, B.; Bilgi, O.; Tuncel, T.; Baloglu, H.; Kandemir E.G. (Istanbul)

Sudden Extramedullary Blast Crisis of Chronic Myeloid Leukemia Manifesting as T-Cell Lymphoblastic Leukemia/Lymphoma Wei, J.; Huang, M.; Wang, Y.; Zhou, J. (Wuhan)

Complete Pathologic Remission of Locally Advanced, Unresectable Pancreatic Cancer (LAPC) after Intensified Neoadjuvant Chemotherapy Hartlapp, I; Müller, J.; Kenn, W.; Steger, U.; Isbert, C.; Scheurlen, M.; Germer, C.-T.; Einsele, H.; Kunzmann, V. (Würzburg)

Management of Cytotoxic Extravasation - ASORS Consensus Recommendations for Diagnosis, Prevention and Treatment de Wit, M. (Berlin); Ortner, P. (München); Lipp, H.-P. (Tübingen); Sehouli, J.; Untch, M.; Ruhnke, M. (Berlin); Mayer-Steinacker, R. (Ulm); Bokemeyer, C. (Hamburg); Jordan, K. (Halle)

\section{Clinical Information}

Single-Source Tumor Doc
for Different Purposes

Ries, M.; Prokosch, H.U. (Erlangen/Nürnberg); Beckmann, M.W.; Bürkle, T. (Erlangen) 